\title{
LARGE-AREA PLASMON-ASSISTED IMAGING OF INDIVIDUAL NANOPARTICLES
}

\author{
Jan BUKÁČEK, Jiří HOMOLA
}

Institute of Photonics and Electronics of the Czech Academy of Sciences, Prague, Czech Republic, EU
bukacek@ufe.cz

https://doi.org/10.37904/nanocon.2019.8768

\begin{abstract}
In the poster a novel approach of a single nanoparticle binding event detection is presented, allowing detection of gold nanoparticles down to $40 \mathrm{~nm}$ diameter. The system is based on a surface plasmon resonance excited on an optical grating combined with a supercontinuum laser and a tuneable filter allowing a use of an optimal spectral region. A large visual field enables a detection of binding events at low concentrations, with a possible application in the field of biochemical analysis.
\end{abstract}

Keywords: Surface plasmon, surface plasmon polariton microscopy, nanoparticles, digital detection

\section{INTRODUCTION}

Surface plasmon resonance microscopy (SPRM) is a method capable of imaging individual metal nanoparticles (NP) binding to metal surface. SPRM allows for imaging individual metal NPs near the metal surface in real time via the scattering of surface plasmons polaritons (SPPs) propagating along the surface [1]. In this work, we report on a new SPRM system based on diffraction gratings (DGs) and explore its potential for the detection of gold NPs.

\section{INSTRUMENTATION}

The optical system for SPRM consists of a light source, collimating optics, a diffraction grating and a detector. The light source is a supercontinuum laser, combined with a tunable filter which generates light of a selected wavelength and $1 \mathrm{~nm}$ spectral width. The emitted light is collimated and made incident on the DG at normal incidence. The gold-coated DG is interfaced with a flow-cell that allows incubation of the surface of DG with liquid samples. The beam reflected from the DG passes through a beam splitter and is focused on the CCD detector by $2 x$ telecentric optics.

Image data are generated in the form of differential images that are created from a current image by subtracting the reference image collected in the beginning of the experiment. Acquisition times varies between 0.2 and 2 $\mathrm{s}$. The imaged area is about $10 \mathrm{~mm}^{2}$ large.

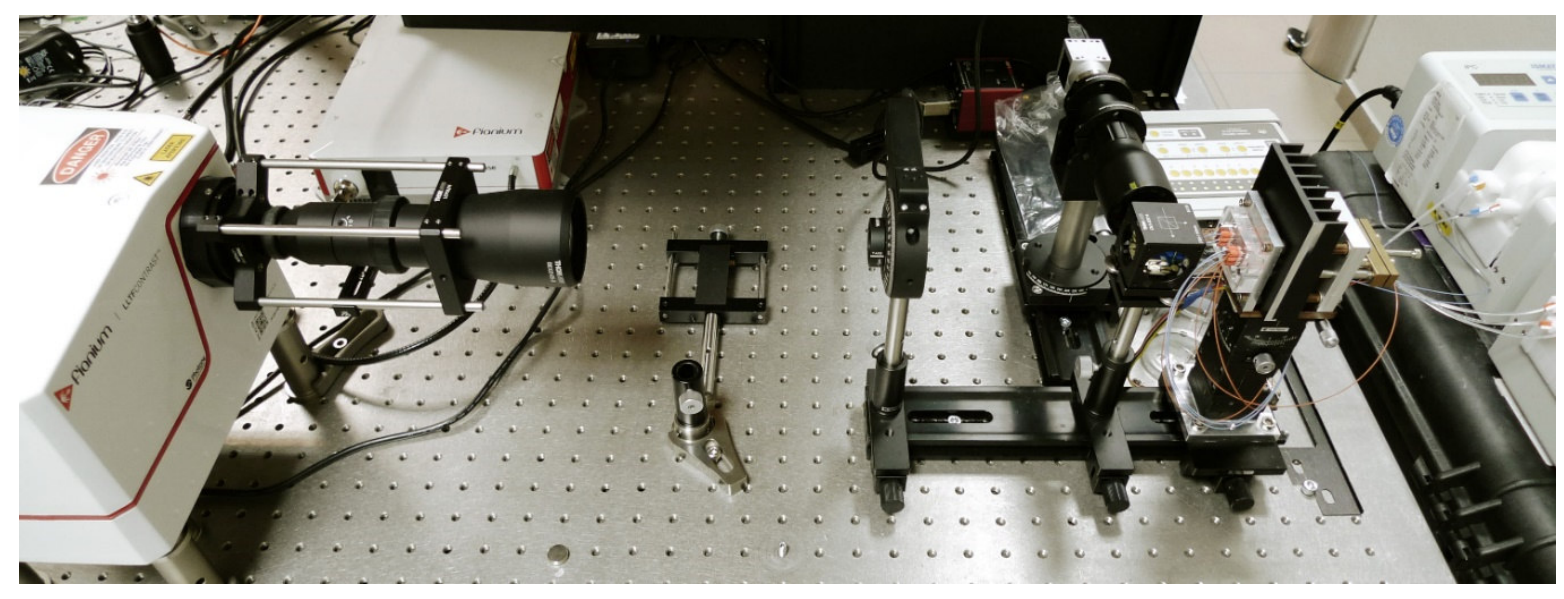




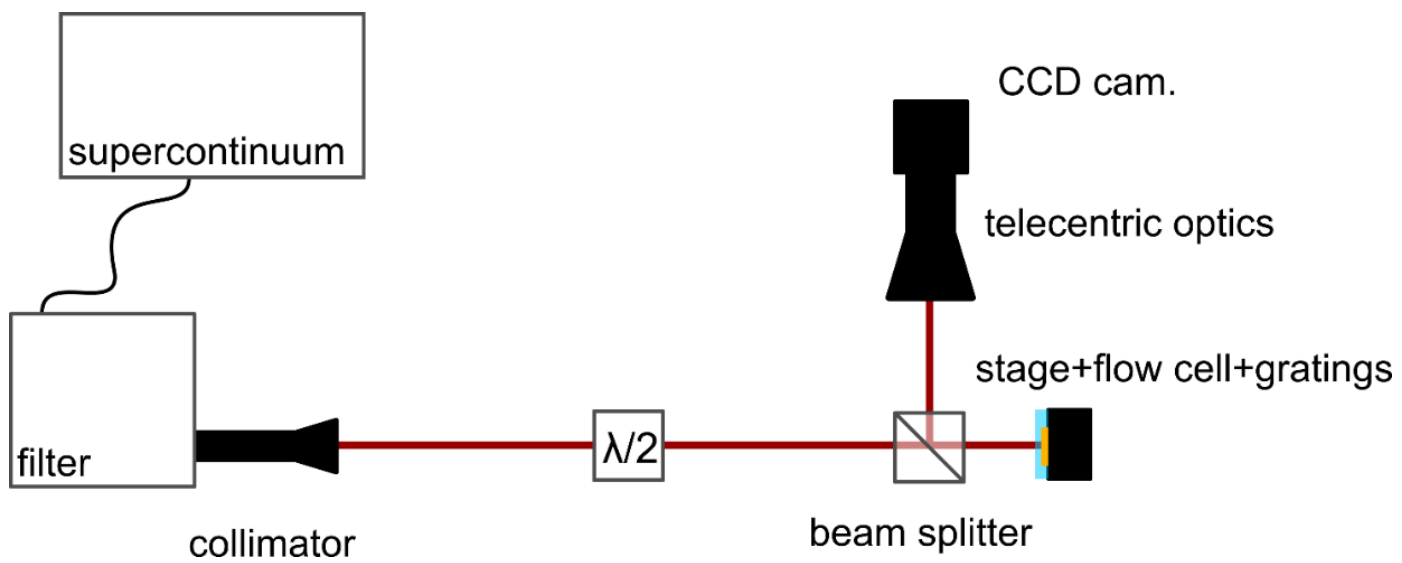

Figure 1 Photograph and scheme of the optical system of SPRM

\section{DIFFRACTION GRATTINGS:}

The DGs were optimized for the highest sensitivity to a refractive index (RI) change in a proximity of the surface. Three spectral regions were chosen, around 750, 650 and $600 \mathrm{~nm}$ (the gratings optimized for these spectral regions are denoted as @750, @650 and @600).

Table 1 Parameters of DGs employed in the system

\begin{tabular}{|l|l|l|l|l|l|}
\hline DG & $\begin{array}{l}\text { DESIGNED } \\
\text { PERIOD [nm] }\end{array}$ & $\begin{array}{l}\text { FABRICATED } \\
\text { PERIOD [nm] }\end{array}$ & $\begin{array}{l}\text { DESIGNED } \\
\text { DEPTH [nm] }\end{array}$ & $\begin{array}{l}\text { FABRICATED } \\
\text { DEPTH [nm] }\end{array}$ & $\begin{array}{l}\text { SENSITIVITY } \\
\text { [RIU-1]* }\end{array}$ \\
\hline$@ 750$ & 527.5 & 405.5 & 40 & 45 & -8.71 \\
\hline$@ 650$ & 453 & 452 & 35 & 32 & 4.51 \\
\hline$@ 600$ & 407.2 & 528.3 & 25 & 24 & 3.21 \\
\hline
\end{tabular}

* The sensitivity is the simulated sensitivity on the RI change of $20 \mathrm{~nm}$-thick layer on the gold surface.

\section{EXPERIMENTAL}

The performance of the SPRM system was characterized in model experiments, where gold NPs were adsorbed on the DG that had been functionalized with a self-assembled monolayer of alkylthiols. SPP resonance wavelength was measured for each DG using SPP spectroscopy and the illumination wavelength for SPRM was determined which yields the best performance (highest image contrast).

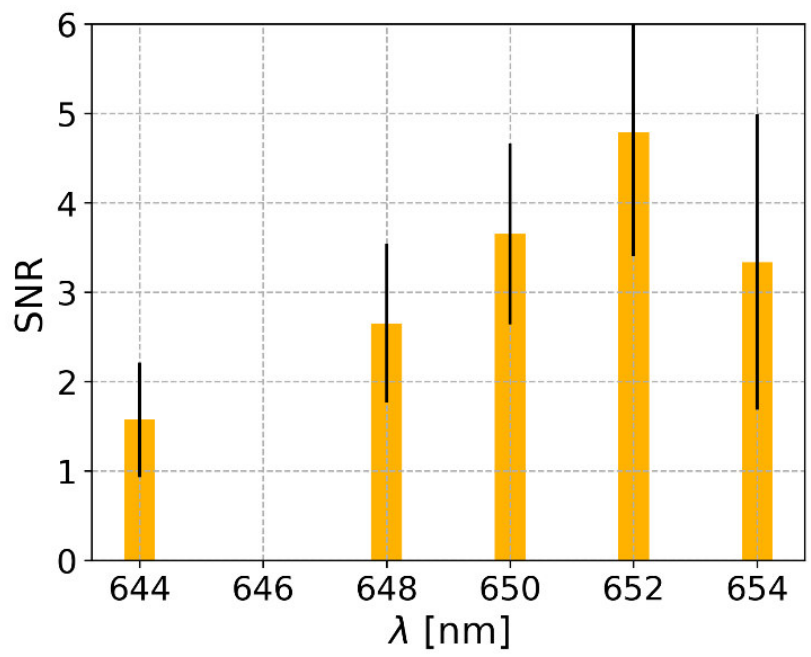

Figure 2 Signal-to-noise ratio as a function of wavelength. DG - @650, NP diameter - 80 nm, dip position - $648 \mathrm{~nm}$ 


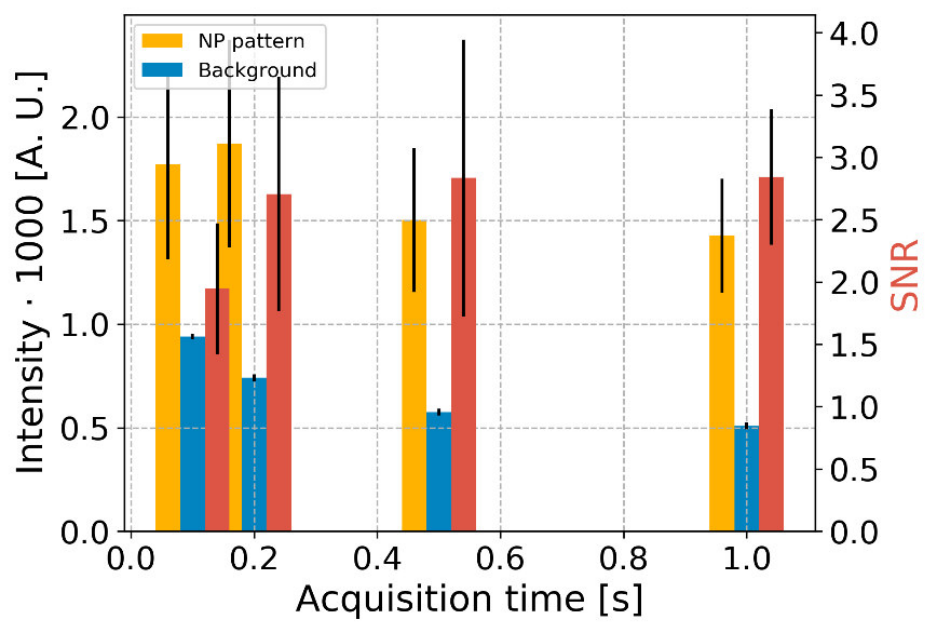

Figure 3 Influence of acquisition time on background intensity, intensity of the NP pattern and SNR. DG - @750, NP diameter $80 \mathrm{~nm}$.

\section{TEMPORAL RESOLUTION:}

Our experiments show that longer acquisition times reduce the noise of the image and improves its contrast (Figure 3). The NPs are considered detectable when the SNR exceeds 2.5.
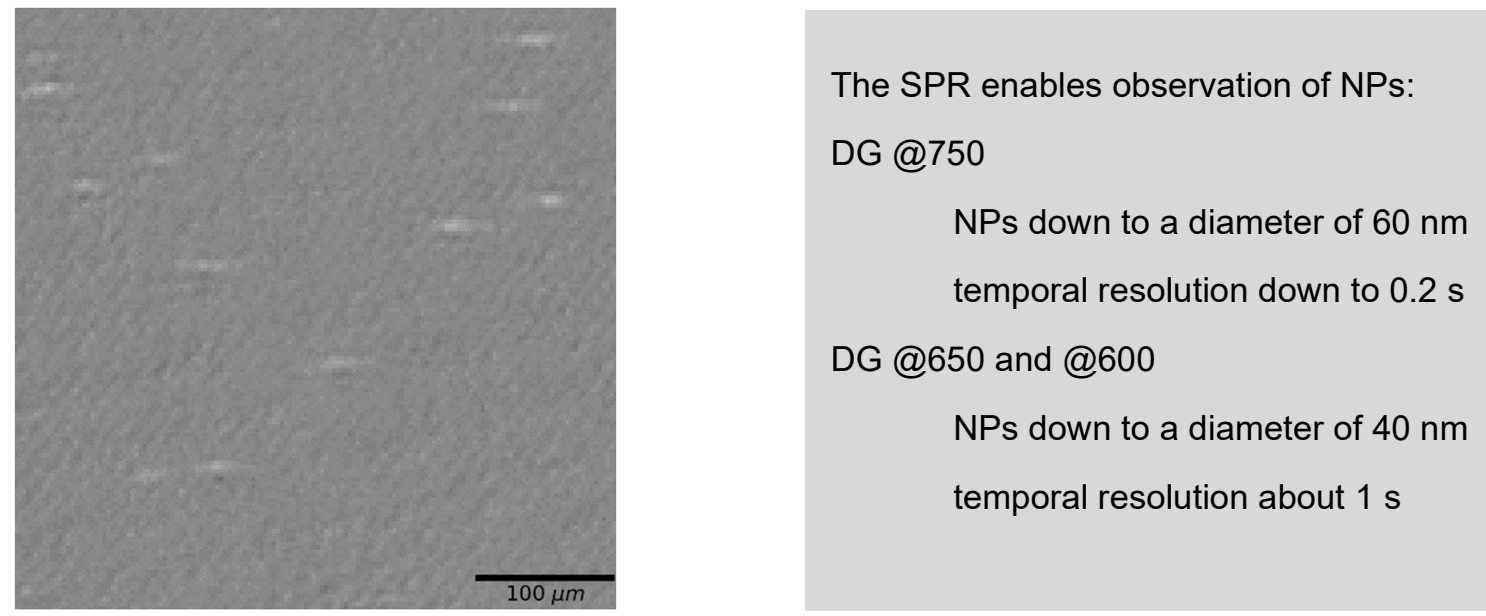

DG @750, NP diameter 80 nm
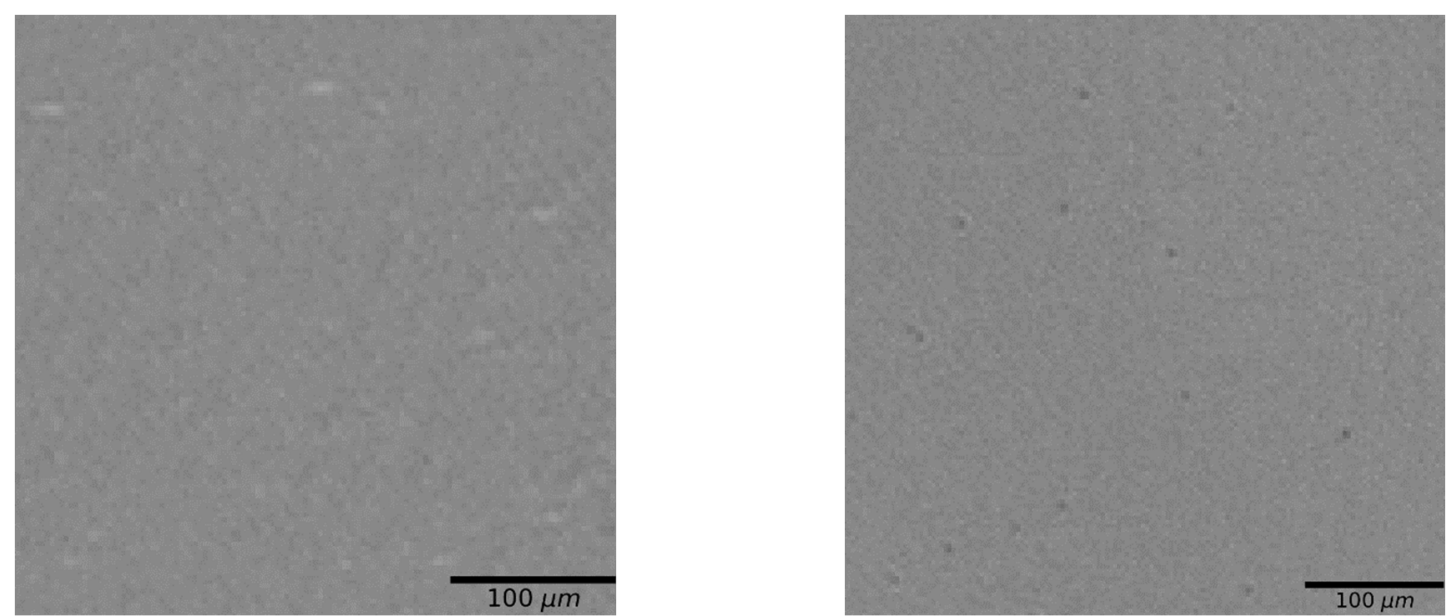

DG @650, NP diameter 40 nm

DG @600, NP diameter 40 nm

Figure 4 Detected patterns of gold NPs. Acquisition time is $1 \mathrm{~s}$ for all the images. 


\section{CONCLUSIONS}

We demonstrate a new SPRM system that allows observing gold NPs down to a diameter of $40 \mathrm{~nm}$ with a temporal resolution down to $0.2 \mathrm{~s}$.

\section{REFERENCE}

[1] A. ZYBIN, Y. KURITSYN, E. GUREVICH, V. TEMCHURA, K. UBERLA, and K. NIEMAX. Real-time detection of single immobilized nanoparticles by surface plasmon resonance imaging. Plasmonics, 5(1):3135, Mar 2010. 